ARTICLE

DOI: $10.1038 / s 41467-017-00466-y$

\title{
Electron paramagnetic resonance microscopy using spins in diamond under ambient conditions
}

\author{
David A. Simpson (10 1,2, Robert G. Ryan ${ }^{3}$, Liam T. Hall (1) 1, Evgeniy Panchenko (1) 1, Simon C. Drew ${ }^{4}$, \\ Steven Petrou $2,4,5,6$, Paul S. Donnelly (1) ${ }^{3}$, Paul Mulvaney (1) ${ }^{3} \&$ Lloyd C.L. Hollenberg (1) 1,2,3,7
}

Magnetic resonance spectroscopy is one of the most important tools in chemical and bio-medical research. However, sensitivity limitations typically restrict imaging resolution to $\sim 10 \mu \mathrm{m}$. Here we bring quantum control to the detection of chemical systems to demonstrate high-resolution electron spin imaging using the quantum properties of an array of nitrogen-vacancy centres in diamond. Our electron paramagnetic resonance microscope selectively images electronic spin species by precisely tuning a magnetic field to bring the quantum probes into resonance with the external target spins. This provides diffraction limited spatial resolution of the target spin species over a field of view of $50 \times 50 \mu \mathrm{m}^{2}$ with a spin sensitivity of $10^{4}$ spins per voxel or $\sim 100 \mathrm{zmol}$. The ability to perform spectroscopy and dynamically monitor spin-dependent redox reactions at these scales enables the development of electron spin resonance and zepto-chemistry in the physical and life sciences.

\footnotetext{
${ }^{1}$ School of Physics, University of Melbourne, Parkville, VIC 3010, Australia. ${ }^{2}$ Centre for Neural Engineering, University of Melbourne, Parkville, VIC 3010, Australia. ${ }^{3}$ School of Chemistry and Bio21 Molecular Science and Biotechnology Institute, The University of Melbourne, Parkville, VIC 3010, Australia. ${ }^{4}$ Department of Medicine, Royal Melbourne Hospital, The University of Melbourne, Parkville, VIC 3010, Australia. ${ }^{5}$ Florey Neuroscience Institute, University of Melbourne, Parkville, VIC 3010, Australia. ${ }^{6}$ Centre for Integrated Brain Function, University of Melbourne, Parkville, VIC 3010, Australia. ${ }^{7}$ Centre for Quantum Computation and Communication Technology, University of Melbourne, Parkville, VIC 3010, Australia. Correspondence and requests for materials should be addressed to D.A.S. (email: simd@unimelb.edu.au) or to L.C.L.H. (email: Iloydch@unimelb.edu.au)
} 
M agnetic resonance spectroscopy techniques have revolutionised detection and imaging capabilities across the life and physical sciences. Electron paramagnetic resonance (EPR), nuclear magnetic resonance (NMR), and magnetic resonance imaging (MRI) are now essential tools in many areas of science and clinical research. Current ambient $\mathrm{NMR}^{1}$ and EPR-based systems ${ }^{2}$ employing field gradients have demonstrated imaging resolution as low as $10 \mu \mathrm{m}$. However, exploring nanoscale biological and chemical processes with sub-micrometre resolution requires a major technological shift.

State-of-the-art cryogenic EPR-based imaging approaches have demonstrated detection from $10^{6}$ spins at sub-micrometre spatial resolution, by reducing the size of the surface loop and scanning, with projected sensitivity down to the 10-100 spins regime $^{3}$. Other high-resolution imaging techniques such as magnetic resonance force microscopy ${ }^{4}$ and scanning tunnelling microscopy provide single-electron spin sensitivity with nanoscale spatial resolution ${ }^{5}$, but they are also constrained to cryogenic temperatures and high vacuum environments which precludes their use in imaging functional biochemical reactions. For EPR applications, the regime of sub-micrometre room temperature spectroscopy and imaging has presented a major challenge. The development of such a technology would provide insights into electron spin dynamics at the nanoscale, including redox dynamics, organic radical formation and complicated transition metal biochemistry at the intra-cellular scale.

Here we report an imaging technique based on quantum probe relaxation (QPR) spectroscopy, which can selectively image spectrally resolved spin targets in aqueous solution with high spatial resolution under ambient conditions. Electron spin detection is achieved using an array of nitrogen vacancy (NV) spin probes in diamond together with wide-field optical microscopy and precise magnetic field alignment as shown in Fig. 1a. As a demonstration of the capabilities, we perform spectroscopic imaging of hexaaqua- $\mathrm{Cu}^{2+}$ complexes, see Fig. $1 \mathrm{~b}$, and their associated redox dynamics over fields of view (FOV) of $\sim 50 \times 50 \mu^{2}$. We demonstrate imaging resolution at the diffraction limit $(\sim 300 \mathrm{~nm})$ with spin sensitivities in the hundreds of zeptomol $\left(10^{-21} \mathrm{~mol}\right)$ range.

\section{Results}

EPR with NV centres in diamond. Electron paramagnetic resonance microscopy is performed by monitoring the spin lattice relaxation times of an array of solid state atomic sized quantum sensors in diamond. The NV probes are engineered at controlled depths $(\sim 6-8 \mathrm{~nm})$ from the diamond surface with an average spatial separation of $\sim 10 \mathrm{~nm}$ via nitrogen ion-implantation (see 'Methods'). Due to the crystal symmetry of diamond, the quantum probes are formed along four possible $\langle 111\rangle$ crystallographic orientations. The NV centre in diamond has a paramagnetic ground-state triplet as shown in Fig. 1c with the $| \pm 1\rangle$ states separated from the $|0\rangle$ state by the diamond zero-field splitting $D=2.87 \mathrm{GHz}$. The degeneracy of the $| \pm 1\rangle$ states is lifted in the presence of a magnetic field and Zeeman split with a gyromagnetic ratio $\gamma_{\mathrm{NV}}=2.8 \mathrm{MHz} \mathrm{G}^{-1}$. The quantum probes can be conveniently spin polarised and their state 'readout' at room temperature under optical excitation at $532 \mathrm{~nm}$. The spin state readout arises from the difference in fluorescence intensity between the $|0\rangle$ and $| \pm 1\rangle$ states, allowing optically detected magnetic resonance (ODMR) of the ground-state magnetic sublevels ${ }^{6}$. In the case of $\mathrm{NV}$ ensembles, the four orientations result in eight possible transitions depending on the applied magnetic field. An external field can be applied along a particular NV symmetry axis and a single microwave $\pi$ pulse used to isolate the subset of NV spin probes which are aligned with the applied magnetic field. The spin lattice relaxation time $\left(T_{1}\right)$ of the quantum probes can be determined by optically polarising the spins into the $m_{\mathrm{s}}=0$ ground state, then allowing them to evolve for a variable time $\tau$, before sampling the spin population with an additional optical pulse. Interactions between the NV probe and a

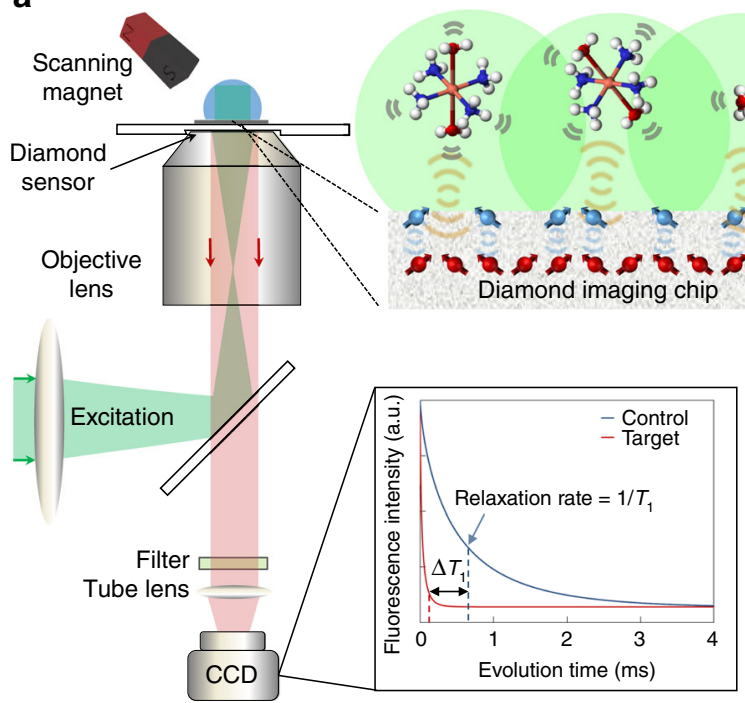

b

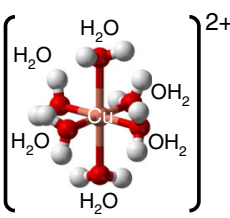

Target-Hexaaqua $\mathrm{Cu}^{2+}$ complex

d
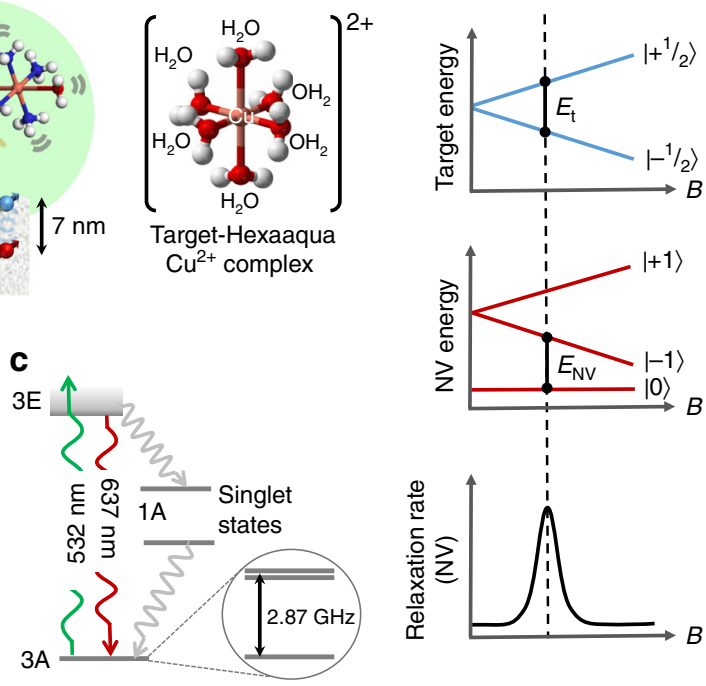

Fig. 1 Quantum probe relaxation microscopy. a Schematic of the microscope system with optical excitation at $532 \mathrm{~nm}$ and resulting NV fluorescence filtered (650-750 nm) and imaged onto an SCMOS camera. Microwave control is provided via a gold microwave resonator evaporated onto a glass coverslip. Expanded view shows the diamond imaging chip with target and surface electronic spins, in addition to the layer of NV centres $7 \mathrm{~nm}$ below the surface. The spin lattice relaxation time, $T_{1}$, across the imaging array is determined from a sequence of fluorescence images, see Fig. 2 for more details. $\mathbf{b}$ Schematic representation of the spin target hexaaqua- $\mathrm{Cu}^{2+}$ complex, $\left[\mathrm{Cu}_{(}\left(\mathrm{OH}_{2}\right)_{6}\right]^{2+}$. c Simplified energy-level diagram for the NV centre highlighting the paramagnetic ground-state triplet. At zero magnetic field the degenerate $|-1\rangle$ and $|+1\rangle$ states are separated from the $|0\rangle$ state by the crystal field splitting of $D=2.87 \mathrm{GHz}$. $\mathbf{d}$ Quantum probe relaxation spectroscopy. Simplified energy diagrams representing the Zeeman splitting for a target spin $\left(\mathrm{Cu}^{2+}\right)$ and the NV probe. When the transition energy, $E_{\mathrm{t}}$, from the target spin $(|-1 / 2\rangle \rightarrow|+1 / 2\rangle)$ is matched to the transition energy, $E_{N V}$, from the NV centre $(|0\rangle \rightarrow|-1\rangle)$, the NV spin relaxation rate $\left(1 / T_{1}\right)$ signal increases 
nearby electronic, nuclear and surface spins species cause the NV net magnetisation to relax from the $m_{\mathrm{s}}=0$ state to a mixture of the three ground triplet states. The $1 / e$ decay time is referred to as the $T_{1}$ time of the probe.

The NV centre ${ }^{7}$ in diamond is a promising system for nanoscale electronic spin detection ideally suited for room temperature biological environments ${ }^{8-10}$. While detection of small numbers of electronic spins (less than 10), based on decoherence $^{11}$, or quantum control protocols ${ }^{12}, 13$ has been carried out using single NV centres ${ }^{13-17}$, and in wide-field array systems ${ }^{18,19}$, spectroscopic microscopy that can target specific electronic spin species is lacking. Spectroscopic detection to date has relied on complex quantum control techniques ${ }^{20}, 21$, which creates major challenges when scaling up to large FOV. The main challenge here is creating a uniform driving field over a wide FOV to address the $\mathrm{NV}$ probes and target spins. We instead employ an optical spectroscopic detection method ${ }^{22}$ based on precise control of a static external magnetic field, $B_{0}$, which is used to tune the NV transition energy into resonance with environmental spins at selected effective $g$-factors. At a given resonance condition the NV and environmental spins (which may comprise intrinsic and target spins) exchange energy resulting in an increase in the measured NV relaxation rate as illustrated in Fig. 1d. This quantum probe relaxation microscopy (QPRM) allows the EPR spectrum of the target spins to be resolved at each imaging pixel.

The relaxation rate, $\Gamma_{1}^{(\mathrm{E})}\left(B_{0}\right)=1 / T_{1}$, of a quantum probe in the presence of an arbitrary magnetic environment (E), comprising intrinsic (I) and/or target (T) spins, is given by the spectral density of the environment $S^{(\mathrm{E})}\left(\omega_{\mathrm{E}}, B_{0}\right)$ convolved with the filter function of the probe, in this case the NV spin:

$$
\Gamma_{1}^{(\mathrm{E})}\left(B_{0}\right)=\int S^{(\mathrm{E})}\left(\omega_{\mathrm{E}}, B_{0}\right) G\left(\Gamma_{2}, \omega_{\mathrm{E}}, B_{0}\right) \mathrm{d} \omega_{\mathrm{E}},
$$

where $G\left(\Gamma_{2}, \omega_{\mathrm{E}}, B_{0}\right)=\frac{b^{2} \Gamma_{2}}{2\left(\Gamma_{2}^{2}+\left(\omega_{\mathrm{NV}}-\omega_{\mathrm{E}}\right)^{2}\right)}$ is the NV filter function given by a Lorentzian dependent on the resonance frequency of environmental spins, $\omega_{\mathrm{E}}$, the coupling strength, $b$, the external magnetic field $B_{0}\left(\omega_{\mathrm{NV}}=\gamma_{\mathrm{NV}} B_{0}\right)$ and $\mathrm{NV}$ transverse relaxation rate $\Gamma_{2}$ (Supplementary Note 1 ). To probe specific components of the environment's spectral density, $S^{(\mathrm{E})}\left(\omega_{\mathrm{E}}, B_{0}\right)$, the $\mathrm{NV}$ filter function can be tuned via the Zeeman interaction and an applied magnetic field, $B_{0}$.

To characterise the inherent magnetic environment of the diamond sensing chip, a calibration experiment is carried out to obtain the relaxation rate spectrum, $\Gamma_{1}^{(1)}\left(B_{0}\right)$ in the absence of a target system. The calibration spectrum reflects the interactions of electronic and nuclear spins both on the surface and within the bulk diamond. The target $(\mathrm{T})$ is then introduced and the NV relaxation rate spectrum corresponding to the combined system, $\Gamma_{1}^{(\mathrm{I}+\mathrm{T})}\left(B_{0}\right)$, mapped. By subtracting the calibration measurement, we obtain the target relaxation spectrum below, see Supplementary Note 1 for details:

$$
\Gamma_{1}^{(\mathrm{T})}\left(B_{0}\right) \approx \Gamma_{1}^{(\mathrm{I}+\mathrm{T})}\left(B_{0}\right)-\Gamma_{1}^{(\mathrm{I})}\left(B_{0}\right),
$$

Given the relatively narrow probe filter function, the target relaxation spectrum is essentially unchanged following deconvolution of Eq. (1) and is therefore identical to the spectral density $S^{(\mathrm{T})}\left(\omega_{\mathrm{E}} ; B_{0}\right)$, up to a normalisation factor.

QPR imaging of hexaaqua- $\mathrm{Cu}^{2+}$ ions. Copper reactions are tightly regulated in biology due to the potential for free copper ions to produce damaging free radical species ${ }^{23-25}$. Therefore, there is significant interest in understanding how $\mathrm{Cu}^{2+}$ and similar transition metal ions interact in varying chemical environments. To date, the majority of techniques used to characterise
$\mathrm{Cu}^{2+}$ ions in biological systems have involved measuring luminescent properties of $\mathrm{Cu}^{2+}$-bound molecular probes ${ }^{26,27}$. Redox reactions of $\mathrm{Cu}^{2+}$ and other transition metals in solution have traditionally been studied using electro-chemical approaches and fluorescent molecular probes that act as surrogate markers of redox status ${ }^{28}$. The QPRM approach described here is label free and non-invasive and does not impact on the function or availability of the $\mathrm{Cu}^{2+}$ complexes. Using spin selective spectroscopy, we can probe redox reaction kinetics in sensing volumes of order attoliters with temporal resolution of order seconds enabling the study of transition metal ions in solution and/or bulk material systems.

We begin with the demonstration of selective detection and spatial imaging of $\mathrm{Cu}^{2+}$ ions. Figure 2a shows the transition frequencies of the NV probe, free electron spins $(\langle g\rangle=2)$ and the target hexaaqua $\mathrm{Cu}^{2+}$ ion $\left(\left\langle g_{\text {eff }}\right\rangle=2.199\right)$ as a function of magnetic field. The electronic configuration of the target spin complex $\left[\mathrm{Cu}\left(\mathrm{OH}_{2}\right)_{6}\right]^{2+}$, is $d^{9}$ with octahedral geometry, giving rise to a single unpaired electron, and an overall electronic spin of 1/2. Degeneracy resulting from Jahn-Teller distortion, see Fig. 1b, gives rise to an axially symmetric Zeeman interaction with a perpendicular and parallel $g$-factor of $g_{\perp}=2.099$ and $g_{\|}=2.400^{29}$, respectively. At room temperature, a weighted average of these two $g$ factors is observed due to motional averaging; when integrated over all possible orientations this results in an isotropic $g$-factor of $\langle g\rangle=1 / 3\left(g_{\|}+2 g_{\perp}\right)=2.199$. From Fig. $2 \mathrm{a}$ the resonance condition for $\mathrm{Cu}^{2+}$ ions $\leftrightarrow \mathrm{NV}$ occurs around $487 \mathrm{G}$. At the resonance point the $\mathrm{Cu}^{2+}$ and $\mathrm{NV}$ probe spins can exchange energy efficiently resulting in a significant reduction in the $\mathrm{NV} T_{1}$ time (increase in the relaxation rate), forming the fundamental QPRM image contrast.

Figure $2 \mathrm{~b}$ presents two $T_{1}$ relaxation curves (measurement sequence shown in the inset) off $(250 \pm 1 \mathrm{G})$ and near resonance $(460 \pm 2 \mathrm{G})$ with $\mathrm{Cu}^{2+}$ spins. The $T_{1}$ time from the $\mathrm{NV}$ probes reduced from $730 \pm 60$ to $96 \pm 1 \mu$ s as the $\mathrm{Cu}^{2+}$ resonance condition was approached. This reduction included a contribution from surface spins, the analysis and subtraction of which is described quantitatively in the next section on spectroscopy. To image the $\mathrm{Cu}^{2+}$ distribution in solution an image mask was fabricated on top of the diamond imaging chip with poly(methyl methacrylate) (PMMA) as described in Fig. 2c (see 'Methods'). A set of single evolution time point, $\tau_{\mathrm{sp}}$, images were obtained at magnetic fields either side of the $\mathrm{Cu}^{2+} \stackrel{\leftrightarrow}{\leftrightarrow}$ NV resonance position. The $\tau_{\mathrm{sp}}$ probe time was set to the $T_{1}$ time obtained from the FOV at each respective magnetic field. This probe time maximised image contrast for small signal changes. The single $\tau_{\mathrm{sp}}$ images were averaged over $N_{\mathrm{c}}=6 \times 10^{6}$ cycles and are shown in Fig. $2 d$.

The QPR image shown in Fig. 2e demonstrates significant image contrast from an effective $2 \mathrm{D}$ distribution of $\mathrm{Cu}^{2+}$ ions defined by the image mask at magnetic fields near the $\mathrm{Cu}^{2+} \leftrightarrow$ $\mathrm{NV}$ resonance condition. This form of imaging is sufficient to spatially image the $\mathrm{Cu}^{2+}$ distribution in solution. In the following section, we perform quantitative spectroscopy on these target spins.

Quantitative electronic spin spectroscopy. To demonstrate quantitative spectroscopy, a thorough understanding of the intrinsic magnetic environment consisting of the surface and intrinsic spins is required before introducing external target spins into the environment. Calibration measurements were conducted with a solution of nitric acid $(4 \mu \mathrm{L}, 1 \mathrm{mM})$, which was used for dissolution of the $\mathrm{Cu}^{2+}$ analyte. The calibration spectrum, $\Gamma_{1}^{(\mathrm{I})}\left(B_{0}\right)$, is shown in Fig. 3a (green diamonds). The strength of the external B-field was determined directly from the Zeeman 
a

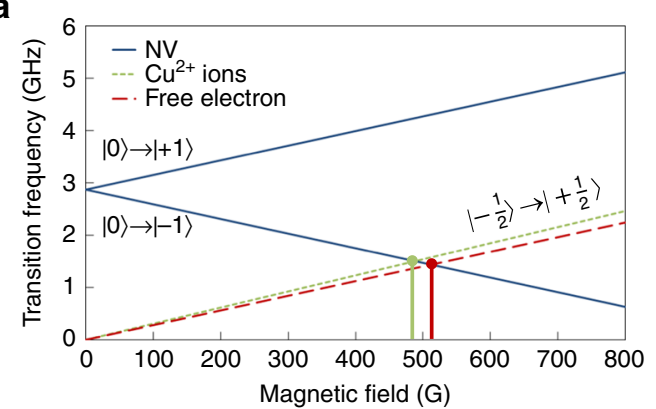

C

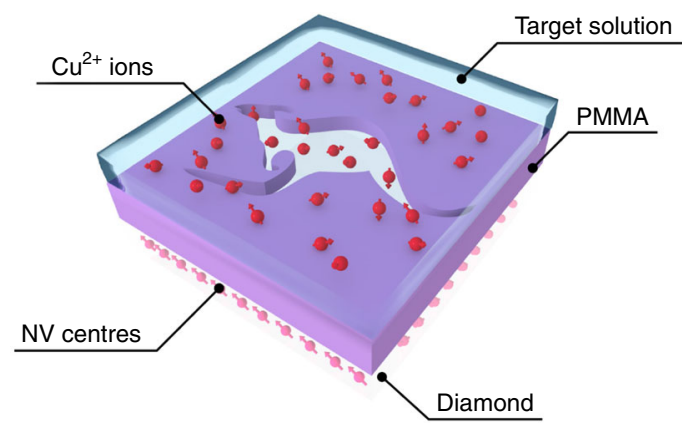

b

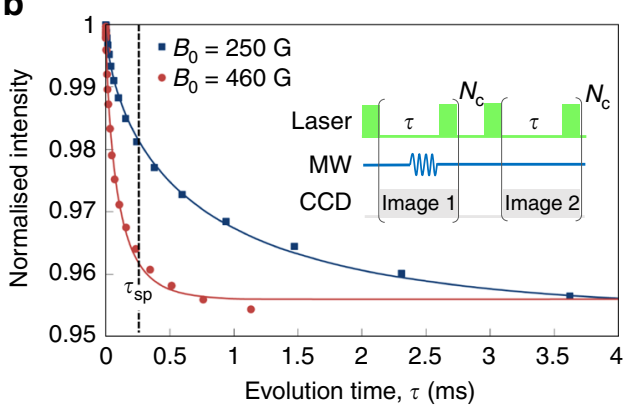

d

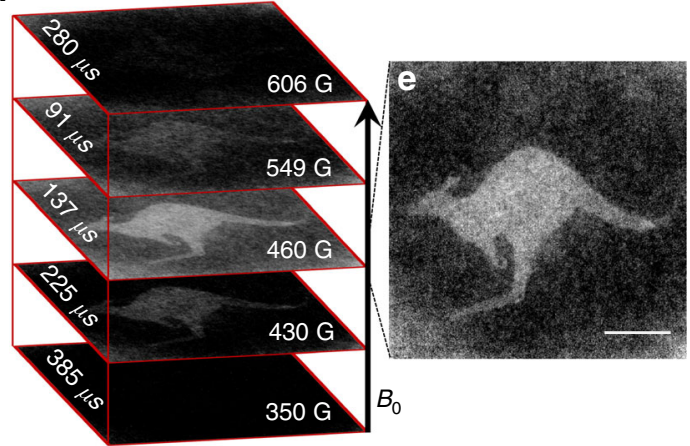

Fig. 2 Quantum probe relaxation imaging of aqueous $\mathrm{Cu}^{2+}$. a Transition frequencies of the hexaaqua- $\mathrm{Cu}^{2+}$ ions and free electrons $(|-1 / 2\rangle \rightarrow|+1 / 2\rangle)$ and $\mathrm{NV}$ spin probes $(|0\rangle \rightarrow|-1\rangle$ and $|0\rangle \rightarrow|+1\rangle)$ as a function of the applied B-field. The solid green line depicts the resonant position of $\mathrm{NV}$ and $\mathrm{Cu}^{2+}(487 \mathrm{G})$, while the solid red line shows the resonance condition for NV and free electron spins ( $512 \mathrm{G}$ ). b $T_{1}$ relaxation curves measured 'near' ( $460 \pm 2 \mathrm{G}$ ) and 'off' ( $250 \pm$ $2 \mathrm{G}$ ) resonance with $\mathrm{Cu}^{2+}$ ions. Inset: $T_{1}$ measurement sequence. Measurements were averaged over $N_{\mathrm{c}}=10,000$ cycles at each ' $\tau$ ' point, with a typical acquisition time of $5 \mathrm{~min}$. c Schematic of the PMMA image template on the diamond imaging chip. $\mathbf{d}$ Single $\tau_{\mathrm{sp}}$ images at various magnetic fields. The measurement sequence for the single $\tau_{\mathrm{sp}}$ images is the same as in $\mathbf{b}$ with $\tau=\tau_{\mathrm{sp}}$. The probe time, $\tau_{\mathrm{sp}}$, at each magnetic field is shown on the left-hand side of the image. Images were averaged over $N_{c}=6 \times 10^{6}$ cycles. The total acquisition time per image was 40 min. e Quantum probe relaxation image showing the spatial distribution of $\mathrm{Cu}^{2+}$ ions near the $\mathrm{Cu}^{2+} \leftrightarrow \mathrm{NV}$ resonance at $460 \mathrm{G}$. The scale bar is $10 \mu \mathrm{m}$

splitting of the $\mathrm{NV}| \pm 1\rangle$ energy states. The magnetic field gradient over the FOV was $<0.4 \%$ (Supplementary Fig. 1). The calibration spectrum was described well by a single Lorentzian fit. The peak at $511.3 \pm 0.5 \mathrm{G}$ corresponds to interactions between the $\mathrm{NV}$ probes and surface electronic spins with an average $\langle g\rangle=2.01 \pm$ 0.02. The half-width of the calibration spectrum $\omega=60.4 \pm 3 \times$ $10^{6} \mathrm{rad} \mathrm{s}^{-1}$ indicates that the dominant contribution to the linewidth is a spin relaxation process on the time scale of tens of ns, consistent with surface spin phonon relaxation being the dominant contribution to the intrinsic spectrum ${ }^{30}$. A surface spin density of 2.4 spins per $\mathrm{nm}^{2}$ can be estimated from the intrinsic spectrum (Supplementary Note 6). Our method thus provides rapid and quantitative spectroscopy of the surface spin noise spectrum and can be used in combination with a variety of surface chemical passivation modes in order to understand and mitigate these effects.

With the intrinsic calibration spectrum $\Gamma_{1}^{(\mathrm{I})}\left(B_{0}\right)$ obtained, quantitative spectroscopy can be performed on the spin target by acquiring the combined $T_{1}$ relaxation rate spectrum $\Gamma_{1}^{(\mathrm{I}+\mathrm{Cu})}\left(B_{0}\right)$. Figure $3 \mathrm{a}$ (blue circles) presents the combined spectrum from a $4 \mu \mathrm{L}(100 \mathrm{mM})$ droplet of $\mathrm{Cu}^{2+}$ solution. The $\mathrm{Cu}^{2+}$ spectrum, $\Gamma_{1}^{(\mathrm{Cu})}\left(B_{0}\right)$ is then obtained by simply subtracting the calibration spectrum as shown in Fig. 3b. The $\mathrm{Cu}^{2+}$ spectrum is de-convolved in Supplementary Note 4 using the measured NV filter function; however, since the width of the filter function $\sim 4 \mathrm{MHz}(\Delta \omega=$ $\left.25.1 \times 10^{6} \mathrm{rad} \mathrm{s}^{-1}\right)$ is significantly less than the half linewidth of the $\mathrm{Cu}^{2+}$ spectrum $\left(\Delta \omega=1.8 \times 10^{9} \mathrm{rad} \mathrm{s}^{-1}\right)$ the relaxation rate spectrum itself represents the spectral density of the hexaaqua$\mathrm{Cu}^{2+}$ environment. For comparison, we have plotted the microwave absorption spectrum obtained from conventional continuous wave (CW)-EPR spectrometer. The plots in Fig. $3 \mathrm{~b}$ were independently normalised to the area under the spectrum and show excellent agreement between the QPRM spectrum from the FOV (red circles) and the conventional CW-EPR spectrum. We emphasise here that the hexaaqua- $\mathrm{Cu}^{2+}$ spectral density is obtained by simply bringing the target spin into resonance, with no active driving of the target spin.

The $\mathrm{Cu}^{2+}$ spectrum can be derived theoretically and is given by the following expression (Supplementary Note 1):

$$
\Gamma_{1}^{(\mathrm{Cu})}\left(B_{0}\right)=\frac{b_{\mathrm{Cu}}^{2}}{2} \frac{\Gamma_{2}^{(\mathrm{I}+\mathrm{Cu})}+R_{\mathrm{Cu}}}{\left(\Gamma_{2}^{(\mathrm{I}+\mathrm{Cu})}+R_{\mathrm{Cu}}\right)^{2}+\left(2 \pi D-\left(\gamma_{\mathrm{Nv}}+\gamma_{\mathrm{Cu}}\right) B_{0}\right)^{2}},
$$

where the $\gamma_{\mathrm{Cu}}$ is the gyromagnetic ratio of the $\mathrm{Cu}^{2+}$ spins, $b_{\mathrm{Cu}}$ characterises the strength of the overall probe-target coupling (Supplementary Note 1 ), and $R_{\mathrm{Cu}}$ is the total width of the spectral density arising from various processes intrinsic to the $\mathrm{Cu}^{2+}$ solution, e.g. intrinsic relaxation $\left(R_{\mathrm{Cu}}^{\mathrm{Relax}}\right)$, dipole-dipole interactions $\left(R_{\mathrm{Cu}}^{\mathrm{dip}}\right)$, spatial $\left(R_{\mathrm{Cu}}^{\mathrm{Spatial}}\right)$ and rotational motion $\left(R_{\mathrm{Cu}}^{\mathrm{Rot}}\right)$. Our analysis shows that the dominant contribution to the linewidth arises from the intrinsic fluctuation rate, i.e. $R_{\mathrm{Cu}} \approx R_{\mathrm{Cu}}^{\mathrm{Relax}}$ which is concentration independent and of order $\mathrm{GHz}$ (see Supplementary Note 2 for further details) ${ }^{29}$.

By fitting Eq. (3) to the $\mathrm{Cu}^{2+}$ spectrum, the depth of the NV probes, $h_{\text {probe, }}$ and the $\mathrm{Cu}^{2+}$ intrinsic fluctuation rate, $R_{\mathrm{Cu}}^{\mathrm{Relax}}$ can be obtained. The fit to the theoretical model, shown in Fig. 3b, is in excellent agreement with the data and yields an estimated probe depth of $h_{\text {probe }}=6.7 \pm 0.04 \mathrm{~nm}$ and $R_{\mathrm{Cu}}^{\mathrm{Relax}}=(1.8 \pm 0.05) \times$ $10^{9} \mathrm{rad} \mathrm{s}^{-1}$. The NV depth is consistent with molecular dynamics simulations ${ }^{31}$. The $\mathrm{Cu}^{2+}$ intrinsic fluctuation rate, $R_{\mathrm{Cu}}^{\mathrm{Relax}}$ is in 
a
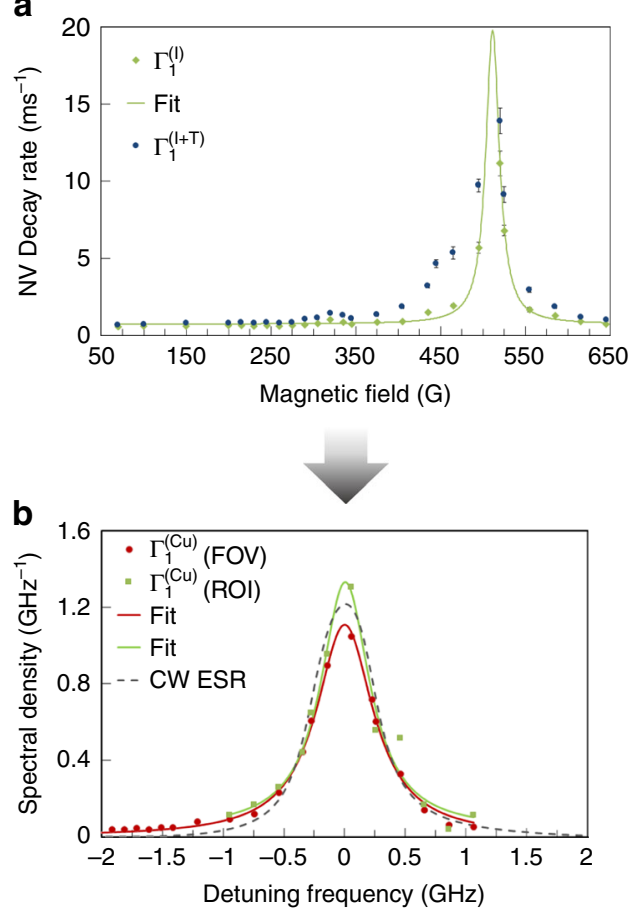

C

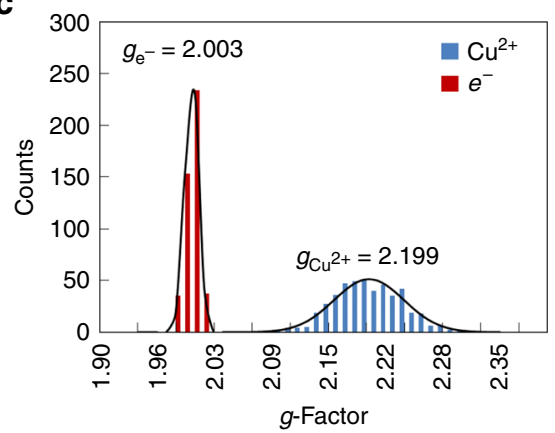

d

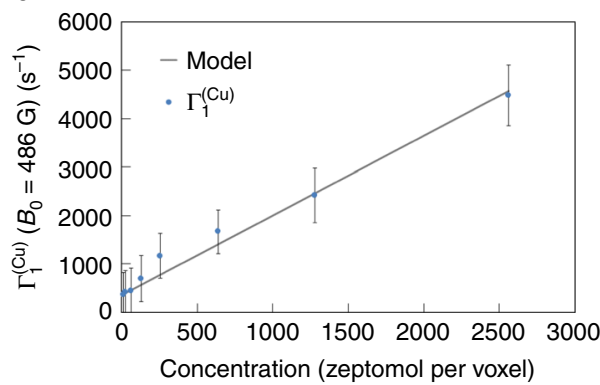

Fig. 3 High-resolution electronic paramagnetic resonance imaging and spectroscopy. a $T_{1}$ decay rate as a function of applied magnetic field $B_{0}$ for $1 \mathrm{mM}$ of nitric acid, (intrinsic-green diamonds) and $100 \mathrm{mM}$ of $\mathrm{Cu}^{2+}$ ions, (intrinsic + target-blue circles). The uncertainty in the relaxation is given by the rms error on the $T_{1}$ stretched exponential fit. b Relaxation rate spectra, $\Gamma_{1}^{(\mathrm{Cu})}$, identified by subtracting $\Gamma_{1}^{(\mathrm{I}+\mathrm{T})}-\Gamma_{1}^{(\mathrm{I})}$ from the field of view $(F O V)\left(35 \times 35 \mu \mathrm{m}^{2}\right)$ and region of interest $(R O I)\left(1.6 \times 1.6 \mu \mathrm{m}^{2}\right)$. The absorption peak at $486 \pm 1 \mathrm{G}$ corresponds to a $g$-factor of $2.21 \pm 0.02$, consistent with the effective $g$-factor for aqueous $\mathrm{Cu}^{2+}$ ions from conventional CW EPR, see dashed grey line. $\mathbf{c}$ Histogram of the measured $g$-factor from each imaging voxel for $\mathrm{Cu}^{2+}$ ions and free electrons. The distribution of $g$-factors for the free electrons is centred at $g=2.003$ with a standard deviation of 0.007 , while the $\mathrm{Cu}^{2+}$ distribution exhibits a distribution centred at $g=2.199$ with a standard deviation of 0.03 . $\mathbf{d}$ Measured relaxation rate, $\Gamma_{1}^{(\mathrm{Cu})}$, on resonance $\left(B_{0}=486 \mathrm{G}\right)$ vs. $\mathrm{Cu}{ }^{2+}$ concentration. The error bars are given by the rms error on the $T_{1}$ stretched exponential fit. The minimum detectable number of spins in a sensing volume defined by $0.025 \mu \mathrm{m}^{3}$ or $(25 \mathrm{aL})$ is $\sim 75,000$ spins per voxel or $\sim 125 \mathrm{zmol}$ per voxel
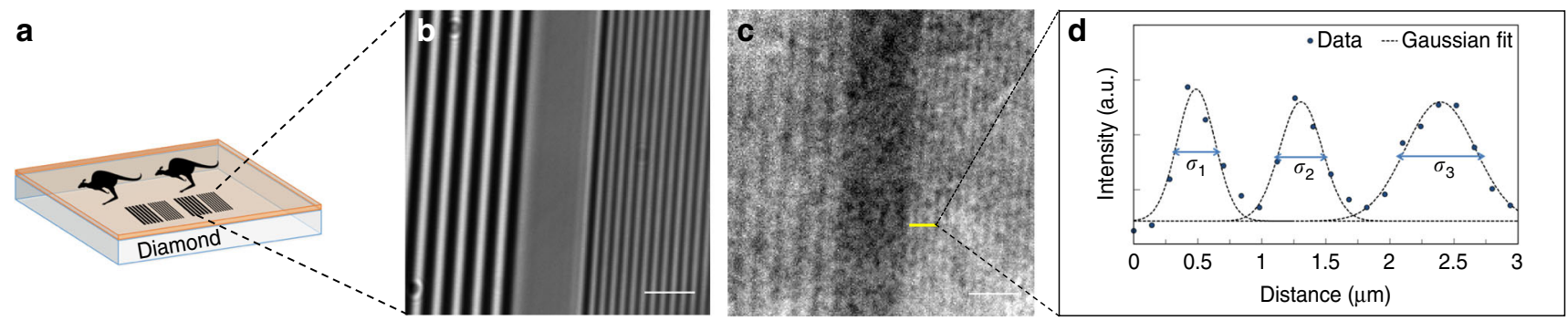

Fig. 4 Spatial resolution of quantum probe relaxation microscopy. a Schematic diagram of the diamond with patterned PMMA layers. b Bright-field image of the grating mask used to determine the image resolution. $\mathbf{b}$ Single $\tau_{\mathrm{sp}}$ image of $\mathrm{Cu}^{2+}$ ions at $460 \mathrm{G}\left(\tau_{\mathrm{sp}}=124 \mu \mathrm{s}, n=12 \times 10^{6}\right)$. The light regions indicate areas of $\mathrm{Cu}^{2+}$. Scale bar in $\mathbf{b}, \mathbf{c}$ is $5 \mu \mathrm{m}$. $\mathbf{d}$ Line cut through a section of the quantum probe relaxation image (yellow line). The Gaussian fit to each peak in the line scan shows linewidths of $\sigma_{1}=290 \pm 30, \sigma_{2}=330 \pm 40$ and $\sigma_{3}=540 \pm 60 \mathrm{~nm}$. The minimum linewidth is consistent with the diffraction limit of the microscope, $\sim 305 \mathrm{~nm}$

excellent agreement with the value obtained from bulk EPR of $R_{\mathrm{Cu}}^{\mathrm{Relax}}=(1.800 \pm 0.005) \times 10^{9} \mathrm{rad} \mathrm{s}^{-1}$, validating our experiment and theoretical approach. The peak position of the spectrum $\left(B_{\text {res }}=486 \pm 1 \mathrm{G}\right)$ is directly related to the $g$-factor of $\mathrm{Cu}^{2+}$ spins by $\left\langle g_{\mathrm{Cu}}\right\rangle=\frac{h D}{\mu_{B} B_{\mathrm{res}}}-g_{N V}$, where $h$ is Planck's constant, $D$ is the zero field splitting of diamond, $\mu_{\mathrm{B}}$ is the Bohr magneton, $g_{\mathrm{NV}}$ is the effective $g$-factor of the NV spin $2.0028^{7}$ and $B_{\text {res }}$ is the magnetic field value corresponding to the peak of the spectrum. From the theoretical fit the measured value of $\left\langle g_{\mathrm{Cu}}\right\rangle=2.21 \pm 0.02$ is in excellent agreement with the literature ${ }^{32}$.

The spectral resolution of our technique is ultimately limited by the point spread function (PSF) of the NV filter function. The width of the PSF is defined by hyperfine and free induction decay mechanisms $\left(\Gamma_{2}\right)$. For the NV array used in this work the PSF is $\sim 4 \mathrm{MHz}$ or $0.14 \mathrm{mT}$. Improvements such as isotopically pure ${ }^{12} \mathrm{C}$ diamond would improve this resolution down to a few hundred $\mathrm{kHz}^{9}$. In the following section, we quantitatively investigate the spectroscopic electronic spin imaging via QPRM.

High-resolution EPR imaging and spectroscopy. The $\mathrm{Cu}^{2+}$ spectrum shown in Fig. 3b was obtained by integrating the signal over the entire FOV at each applied magnetic field. To investigate the spectroscopic spatial resolution of the QPRM we performed a separate experiment, and interrogated a subset of pixels. The goal was to determine the $T_{1}$ relaxation rates of the $\mathrm{NV}$ probes from a 
a

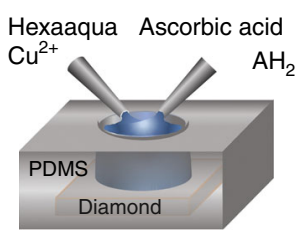

b

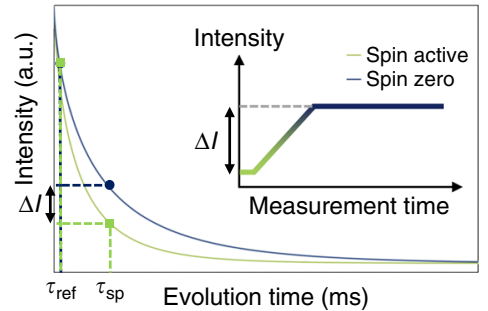

C

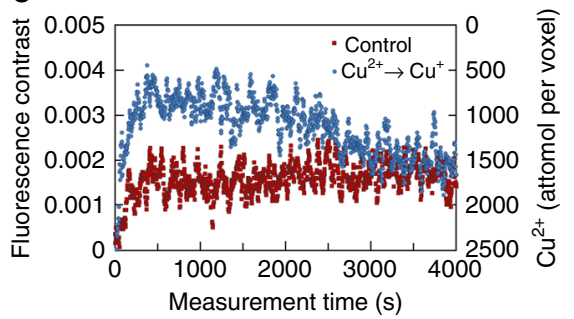

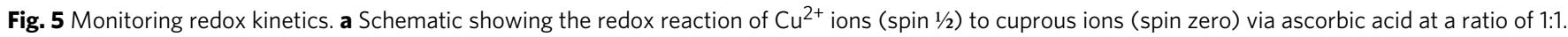
b Dynamic quantum probe relaxation microscopy using a two point $T_{1}$ measurement scheme to detect redox reactions of spin active molecules. The first time point, $\tau_{\text {ref, }}$ is used to normalise the fluorescence signal over the entire FOV $(50 \times 50 \mu \mathrm{m})$ with the second time point $\tau_{\mathrm{sp}}$ set to the $T_{1}$ time of the $\mathrm{NV}$ probes. By monitoring the normalised intensity over time, changes in the $T_{1}$ rate will manifest as a change in the fluorescence intensity as shown schematically in the inset of b. c Two-point $T_{1}$ measurement showing the control experiment (red squares) consisting of a dilution of the Cu ${ }^{2+}$ from 100 mM to $50 \mathrm{mM}$ with water and the reduction of $\mathrm{Cu}^{2+}$ using ascorbic acid (blue circles). The $\mathrm{Cu}^{+}$is found to disproportionate/re-oxidise over a period of $1 \mathrm{~h}$. The fluorescence intensity was determined from the FOV at measurement intervals of $3 \mathrm{~s}$

target sensing volume defined by the imaging pixel size and probe depth. The $\mathrm{Cu}^{2+}$ spectrum obtained from a region of interest (ROI) of $1.6 \times 1.6 \mu \mathrm{m}^{2}$ is shown in Fig. $3 \mathrm{~b}$ (green circles) and is in excellent agreement with the spectrum obtained over the entire FOV. To investigate the uniformity of the measured signal across the imaging area we plot the measured $g$-factor from each ROI pixel as a histogram in Fig. 3c. The distribution is uniform over the imaging area with a mean of $\left\langle g_{\mathrm{Cu}}\right\rangle=2.19 \pm 0.03$, consistent with the results for the FOV. A similar procedure was conducted for the calibration spectrum; this yielded a narrow distribution centred at $\left\langle g_{\text {surface }}\right\rangle=2.003 \pm 0.007$ consistent with the $g$-factor for free electrons (Fig. 3c).

At this point, we quantify the number of detected spins per sensing volume. The ROI sensing voxel is defined by $1.6 \times$ $1.6 \times 0.01 \mu \mathrm{m}^{3}\left(0.025 \mu \mathrm{m}^{3}\right)$ or $25 \mathrm{aL}$ (Supplementary Note 5). For $100 \mathrm{mM} \mathrm{Cu}^{2+}$ this equates to the detection of $1.5 \times 10^{6} \mathrm{Cu}^{2+}$ spins or $\sim 2$ amol per voxel. This is by no means the detection limit. To determine the sensitivity of the system the concentration of $\mathrm{Cu}^{2+}$ ions in solution was varied whilst on resonance with the NV probes, as shown in Fig. 3d. Using the NV probe height and intrinsic $\mathrm{Cu}^{2+}$ fluctuation rate, $R_{\mathrm{Cu}}^{\text {Relax }}$ obtained from the $\mathrm{Cu}^{2+}$ spectrum (FOV) we can fit the concentration data using the following expression (Supplementary Note 1, Supplementary Eq. (11)):

$$
\mathrm{Cu}^{2+}=\frac{4.35 \times 10^{10} \cdot \Gamma_{1}^{(\mathrm{Cu})}\left(B_{\mathrm{res}}\right) \cdot h_{\mathrm{probe}}^{3}\left(\Gamma_{2}^{(\mathrm{I}+\mathrm{Cu})}+R_{\mathrm{Cu}}^{\mathrm{Relax}}\right)^{2}}{\left(\Gamma_{2}^{(I+\mathrm{Cu})}+R_{\mathrm{Cu}}^{\mathrm{Relax}}\right)} \mathrm{moll}^{-1},
$$

where $\Gamma_{1}^{(\mathrm{Cu})}\left(B_{\text {res }}\right)$ is the decay rate from the $\mathrm{Cu}^{2+}$ spins measured on resonance, $h_{\text {probe }}=6.7 \mathrm{~nm}, \Gamma_{2}^{(\mathrm{I}+\mathrm{Cu})}=4 \mathrm{MHz}$ and $R_{\mathrm{Cu}}^{\mathrm{Relax}}=$ $1.8 \times 10^{9} \mathrm{rad} \mathrm{s}^{-1}$. The minimal detectable concentration was determined from the point at which the $T_{1}$ measurement uncertainty was equivalent to the measured change in $T_{1}$ in Fig. 3d. This corresponds to a minimum detectable number of spins for a single voxel of $\sim 75,000$ or $\sim 125 \mathrm{zmol}$. To date, ambient EPR systems exhibit spin sensitivities of order $2 \times 10^{8} \mathrm{G}^{-1} \mathrm{~Hz}^{-1 / 2} 33,34$. Given our intrinsic linewidth of $4 \mathrm{MHz}$ $(1.4 \mathrm{G})$ our technique represents a $10^{4}$ improvement in spin sensitivity.

To quantify the ultimate spatial imaging resolution, we move to a separate region of the imaging chip with an image mask comprised of a series of gratings of width $\sim 500 \mathrm{~nm}$ and pitch of $1 \mu \mathrm{m}$ as shown in Fig. 4a. The QPR image at $460 \mathrm{G}$ shown in Fig. $4 \mathrm{~b}$ reveals the $\mathrm{Cu}^{2+}$ spatial distribution. A line cut through a series of grating lines is shown in Fig. 4c, demonstrating an imaging resolution of $290 \pm 30 \mathrm{~nm}$ which is in excellent agreement with the optical resolution of the microscope $1.22 \lambda$ / $(2 \mathrm{NA}) \approx 305 \mathrm{~nm}$.
These results show that spatial imaging of $\mathrm{Cu}^{2+}$ ions with diffraction limited resolution and zeptomol sensitivity can be achieved under ambient conditions.

Monitoring redox reactions kinetics. Finally, we demonstrate dynamic spin detection by monitoring the redox state of $\mathrm{Cu}^{2+}$ ions in the presence of a reducing agent, ascorbic acid $\left(\mathrm{AH}_{2}\right)$ as described in Fig. 5a. To capture dynamic redox changes, we implement a two-point $T_{1}$ measurement scheme with an external magnetic field set to $\mathrm{Cu}^{2+} \leftrightarrow \mathrm{NV}$ resonant point $B_{0}=486 \mathrm{G}$ (Fig. 5b). The reaction kinetics for $\mathrm{Cu}^{2+}$ in the presence of ascorbic acid can be described by an anaerobic chain-beginning reaction $2 \mathrm{Cu}^{2+}+\mathrm{AH}_{2} \leftrightarrow 2 \mathrm{Cu}^{+}+\mathrm{A}+2 \mathrm{H}^{+}$. The low value of the equilibrium constant, $K_{\mathrm{e}}=(5 \pm 2) \times 10^{-9} \mathrm{M}^{2} 35$, suggests that after the initial reduction to $\mathrm{Cu}^{+}$the $\mathrm{Cu}^{+}$re-oxidises back to $\mathrm{Cu}^{2+}$ which dominates at long times, consistent with the expected disproportionation of $\mathrm{Cu}^{+}$ions.

Figure $5 \mathrm{c}$ (red squares) shows a control measurement performed by diluting the $\mathrm{Cu}^{2+} 100 \mathrm{mM}$ solution by $1: 1$ with MilliQ water. The integrated fluorescence intensity from the FOV was recorded at $3 \mathrm{~s}$ intervals over $4000 \mathrm{~s}$. The $t=0$ point represents the time MilliQ water was added. The fluorescence intensity change $(0.2 \%)$ is consistent with the change in $T_{1}$ decay expected when the $\mathrm{Cu}^{2+}$ concentration is reduced from 100 to 50 $\mathrm{mM}$. The short time oscillations observed in the control curves arise from fluctuations in the laser excitation intensity. These fluctuations do not impact the long-term reduction and oxidation trends in the data and can be reduced using active feedback on the laser modulation. The measured fluorescence intensity is proportional to the $\mathrm{Cu}^{2+}$-induced decay rate and is given by Eq. (5), see Supplementary Note 7 for details:

$$
\Gamma_{1}^{(\mathrm{Cu})}\left(B_{\mathrm{res}}\right)=\frac{\Delta I}{c \tau_{\mathrm{sp}} \Delta I_{0}},
$$

where $\tau_{\mathrm{sp}}$ is the measurement time point in the two-point $T_{1}$ measurement sequence, $\frac{\Delta I}{\Delta I_{0}}$ is the percentage change in fluorescence intensity and $c=0.04$ is the spin relaxation contrast governed by the NV ensemble.

Equations (4) and (5) can be used to translate the change in fluorescence intensity into the $\mathrm{Cu}^{2+}$ concentration. This allows the $\mathrm{Cu}^{2+}$ concentration per voxel to be determined over time as shown in Fig. 5c. To investigate dynamic redox reactions, we introduce ascorbic acid $\left(\mathrm{AH}_{2}\right)$ at a ratio of $1: 1$ with $\mathrm{Cu}^{2+}$ ions. Figure $5 \mathrm{c}$ (blue squares) shows the two point $T_{1}$ measurement from the FOV over the same time period as the control. 
The ascorbic acid was found to reduce $\sim 60 \%$ of the $\mathrm{Cu}^{2+}$ ions in the FOV within $500 \mathrm{~s}$. The reduced spin-zero cuprous ions are not stable in solution and slowly convert back into $\mathrm{Cu}^{2+}$ via disproportionation and/or aerobic oxidation after $4000 \mathrm{~s}$. The final intensity returns to that of the diluted control concentration. This demonstration shows that the redox kinetics from sensing volumes of $\sim 25 \mathrm{fL}$ can be monitored over time and with a sensitivity of order attomol.

\section{Discussion}

In summary, we have demonstrated a QPRM using an array of NV probes in diamond. The microscope operates at room temperature and in ambient conditions and allows simple non-invasive spectroscopy of unpaired electron spin systems in aqueous solution and under biologically compatible conditions. In demonstrating the system, we have focused on the spectroscopy and imaging of the hexaaqua- $\mathrm{Cu}^{2+}$ complex in aqueous solution. We have demonstrated species-specific spatial imaging of the spin target with diffraction limited resolution at $300 \mathrm{~nm}$ and ultimate spin sensitivity approaching the zeptomol level. We have shown how quantitative spectroscopic imaging can be performed on external $\mathrm{Cu}^{2+}$ spins in sensing volumes down to 25 aL with micrometre spatial resolution. The theoretical framework describing the interaction of the target spin system and the $\mathrm{NV}$ spin probe is in excellent agreement with the experimental data.

At present, the transition metal detection levels in these initial demonstrations are several orders of magnitude higher than those found in typical biological environments. However, straightforward improvements in material design of the diamond imaging chip will dramatically reduce the acquisition time and improve the sensitivity towards unpaired electron spins. For example improving the conversion efficiency of NV centres in our imaging chip from 1 to $10 \%^{36}$ combined with photon collection efficiency gains ${ }^{37}$ would see a factor of 20 improvement in the fluorescence signal which would allow sub-second acquisition times and redox kinetics from just tens of zeptomoles of analyte. Furthermore, improved surface passivation of the diamond to remove unwanted surface electron spins will bring an additional order of magnitude improvement in spin sensitivity ${ }^{38}$.

The application of quantum control to the detection and imaging of electronic spin systems represents a significant step forward. The work reported here demonstrates that quantum sensing systems can accommodate the fluctuating Brownian environment encountered in 'real' chemical systems and the inherent fluctuations in the spin environment of ions undergoing ligand rearrangement. A key feature of the technique is the high sensitivity achieved by virtue of the high axial resolution. There are a variety of approaches which can be used to bring systems of interest into close proximity with the diamond imaging chip including chemical functionalisation routes which are now well established for diamond ${ }^{39}$ or, in the case of cell membrane systems, targets may be formed or adhered directly on the surface of the diamond ${ }^{15}$. Therefore, QPRM represents a promising approach for probing fundamental nanoscale biochemistry such as binding events ${ }^{40}$ catalytic reactions, electron transfer processes and the intra-cellular transition metal concentration in the periplasm of prokaryotic cells ${ }^{41}$.

\section{Methods}

Materials. The diamond imaging sensor used in this work is engineered from electronic grade Type IIa diamond (Element 6 ). The diamonds were thinned, cut and re-polished to a $2 \times 2 \times 0.1 \mathrm{~mm}^{3}$ crystal (DDK, USA). NV defects were engineered via ion implantation of ${ }^{15} \mathrm{~N}$ atoms at an energy of $4 \mathrm{keV}$ and dose of $1 \times 10^{13}$ ions per $\mathrm{cm}^{2}$. Molecular dynamic simulations indicate a NV depth range between 5 and $10 \mathrm{~nm}^{31}$ which is consistent with the depth measurement from our analysis of $6.7 \mathrm{~nm}$. The implanted sample was annealed at $1000^{\circ} \mathrm{C}$ for $3 \mathrm{~h}$ and acid treated to remove any unwanted surface contamination. The density of $\mathrm{NV}$ centres post annealing was $1 \times 10^{11} \mathrm{NV}$ per $\mathrm{cm}^{2}$. The linewidth of the ODMR was typically $4 \mathrm{MHz}$. The hyperfine spectrum from the ${ }^{15} \mathrm{~N}$ implant could not be resolved due to the inhomogeneous broadening from the dipole coupling of ${ }^{13} \mathrm{C}$ spins present in the material at a concentration of $1.1 \%$. The typical $T_{1}$ time of the array was $1.8 \mathrm{~ms}$ off resonance with a de-phasing time $T_{2}$ of $\sim 2 \mu$ s.

Optical imaging. The wide-field imaging was performed on a modified Nikon inverted microscope (Ti-U). Optical excitation from a $532 \mathrm{~nm}$ Verdi laser was focused $(f=300 \mathrm{~mm}$ ) onto an acousto-optic modulator (Crystal Technologies Model 3520-220) and then expanded and collimated (Thorlabs beam expander GBE05-A) to a beam diameter of $10 \mathrm{~mm}$. The collimated beam was focused using a wide-field lens $(f=300 \mathrm{~mm})$ to the back aperture of the Nikon $\times 60(1.4 \mathrm{NA})$ oil immersion objective via a Semrock dichroic mirror (Di02-R561-25 $\times 36$ ). The NV fluorescence was filtered using two bandpass filters before being imaged using a tube lens $(f=300 \mathrm{~mm})$ onto a sCMOS camera (Neo, Andor). Microwave excitation to drive the NV spin probes was applied via an omega gold resonator (diameter $=$ $0.8 \mathrm{~mm}$ ) lithographically patterned onto a glass coverslip directly under the diamond imaging chip. The microwave signal from an Agilent microwave generator (N5182A) was switched using a Miniciruits RF switch (ZASWA-2-50DR+). The microwaves were amplified (Amplifier Research 20S1G4) before being sent to the microwave resonator. A Spincore Pulseblaster (ESR-PRO $500 \mathrm{MHz}$ ) was used to control the timing sequences of the excitation laser, microwaves and sCMOS camera and the images where obtained and analysed using custom LabVIEW code. The excitation power density used for imaging was $30 \mathrm{~W}$ per $\mathrm{mm}^{2}$ and all images were taken in an ambient environment at room temperature.

Image mask. The fabrication of the image mask was done by cleaning the diamond imaging chip in hot acetone $\left(65^{\circ} \mathrm{C}\right)$ followed by rinsing in isopropanol alcohol (IPA) and deionised water. The sample was then spin-coated with $280 \mathrm{~nm}$ thick PMMA A4 950k resist. After the spin-coating the sample was baked on the hot plate for $15 \mathrm{~min}$ at $100{ }^{\circ} \mathrm{C}$ followed by additional $10 \mathrm{~min}$ at $170{ }^{\circ} \mathrm{C}$. This enables a better solvent evaporation and prevents the resist from outgassing in vacuum. A 30 $\mathrm{nm}$ thick conduction layer of $\mathrm{Cr}$ was deposited on the resist at the rate of $0.2 \mathrm{~A} \mathrm{~s}^{-1}$. The conduction layer provides charge dissipation during the electron beam lithography (EBL) exposure. The sample was exposed to create a desired pattern using a $100 \mathrm{keV}$ EBPG5000+EBL system. The exposed resist was then developed in a mixture of methyl isobutyl ketone (MIBK) and IPA in the ratio of 1:3. The development was performed for $1 \mathrm{~min}$ and the sample was then rinsed in a fresh IPA and deionised water.

Image analysis. Image analysis was performed using custom LabVIEW code. The $T_{1}$ relaxation images were obtained by determining the $T_{1}$ decay curve at each pixel and fitting the data to a stretched exponential of the form $y=A \exp \left(\left(t / T_{1}\right)^{p}\right)+c$, where $A$ is the amplitude of the exponential decay, $T_{1}$ is the spin lattice relaxation time, $p$ is the stretched exponential power $(p=1$ represents a single exponential decay) and $c$ is the offset. Near surface NV defects are known to exhibit a distribution of $T_{1}$ times from the NV ensemble depending on their proximity to the surface and local spin environment ${ }^{18}$. This distribution leads to a non-exponential $T_{1}$ decay which is characterised well by a stretched exponential function. The amplitude and offset are left as free parameters since these can vary depending on the alignment of the magnetic field particularly around the excited-state level anti-crossing (ESLAC) at $512 \mathrm{G}$.

Magnetic field alignment. Magnetic field alignment was achieved by monitoring the NV fluorescence signal near the ESLAC as described in ref. ${ }^{42}$. The fluorescence at $512 \mathrm{G}$ was extremely sensitive to the field alignment and could be aligned with a particular axis to a precision better than $0.1^{\circ}$. The magnetic field gradient is characterised across the FOV by determining the Zeeman splitting at each pixel via ODMR (Supplementary Note 3). The measured field gradient in the $y$ direction was $0.4 \%$ and $0.07 \%$ in the $x$ direction.

Continuous wave EPR. Continuous-wave EPR spectra were acquired using a CMS8400 X-band $(9.4 \mathrm{GHz})$ spectrometer (Adani, Belarus) fitted with a TE102 cavity and operating at a fixed receiver time constant of $100 \mathrm{~ms}$ and $100 \mathrm{kHz}$ magnetic field modulation. Solution measurements were made at room temperature using a quartz flat cell (Wilmad, WG-808-Q).

Data availability. The data that support the findings of this study are available from the corresponding author upon reasonable request.

Received: 10 February 2017 Accepted: 30 June 2017

Published online: 06 September 2017 


\section{References}

1. van der Kolk, A. G., Hendrikse, J., Zwanenburg, J. J. M., Visser, F. \& Luijten, P. R. Clinical applications of 7 T MRI in the brain. Eur. J. Radiol. 82, 708-718 (2013).

2. Deng, Y. et al. Fast 3D spatial EPR imaging using spiral magnetic field gradient. J. Magn. Reson. 185, 283-290 (2007).

3. Artzi, Y., Twig, Y. \& Blank, A. Induction-detection electron spin resonance with spin sensitivity of a few tens of spins. Appl. Phys. Lett. 106, 084104 (2015).

4. Rugar, D., Budakian, R., Mamin, H. \& Chui, B. Single spin detection by magnetic resonance force microscopy. Nature 430, 329-332 (2004).

5. Baumann, S. et al. Electron paramagnetic resonance of individual atoms on a surface. Science 350, 417-420 (2015)

6. Gruber, A. et al. Scanning confocal optical microscopy and magnetic resonance on single defect centers. Science 276, 2012-2014 (1997).

7. Doherty, M. W. et al. The nitrogen-vacancy colour centre in diamond. Phys. Rep. 528, 1-45 (2013).

8. Le Sage, D. et al. Optical magnetic imaging of living cells. Nature 496, 486-489 (2013).

9. Glenn, D. R. et al. Single-cell magnetic imaging using a quantum diamond microscope. Nat. Methods 12, 736-738 (2015).

10. McGuinness, L. P. et al. Quantum measurement and orientation tracking of fluorescent nanodiamonds inside living cells. Nat. Nanotechnol. 6, 358-363 (2011).

11. Cole, J. H. \& Hollenberg, L. C. L. Scanning quantum decoherence microscopy. Nanotechnology 20, 495401 (2009).

12. Lovchinsky, I. et al. Nuclear magnetic resonance detection and spectroscopy of single proteins using quantum logic. Science 351, 836-841 (2016).

13. Shi, F. et al. Single-protein spin resonance spectroscopy under ambient conditions. Science 347, 1135-1138 (2015)

14. McGuinness, L. P. et al. Ambient nanoscale sensing with single spins using quantum decoherence. New J. Phys. 15, 073042 (2013).

15. Kaufmann, S. et al. Detection of atomic spin labels in a lipid bilayer using a single-spin nanodiamond probe. Proc. Natl Acad. Sci. 110, 10894-10898 (2013).

16. Sushkov, A. O. et al. All-optical sensing of a single-molecule electron spin. Nano Lett. 14, 6443-6448 (2014).

17. Ermakova, A. et al. Detection of a few metallo-protein molecules using color centers in nanodiamonds. Nano Lett. 13, 3305-3309 (2013).

18. Steinert, S. et al. Magnetic spin imaging under ambient conditions with sub-cellular resolution. Nat. Commun. 4, 1607 (2013).

19. Ziem, F. C., Götz, N. S., Zappe, A., Steinert, S. \& Wrachtrup, J. Highly sensitive detection of physiological spins in a microfluidic device. Nano Lett. 13, 4093-4098 (2013).

20. Loretz, M. et al. Spurious harmonic response of multipulse quantum sensing sequences. Phys. Rev. X 5, 021009 (2015).

21. DeVience, S. J. et al. Nanoscale NMR spectroscopy and imaging of multiple nuclear species. Nat. Nanotechnol. 10, 129-134 (2015).

22. Hall, L. T. et al. Detection of nanoscale electron spin resonance spectra demonstrated using nitrogen-vacancy centre probes in diamond. Nat. Commun. 7, 10211 (2016).

23. Letelier, M. E. et al. Possible mechanisms underlying copper-induced damage in biological membranes leading to cellular toxicity. Chem. Biol. Interact. 151, 71-82 (2005)

24. Goldstein, S. \& Czapski, G. The role and mechanism of metal ions and their complexes in enhancing damage in biological systems or in protecting these systems from these systems from the toxicity of $\mathrm{O}_{2}{ }^{-}$. J. Free Radic. Biol. Med. 2, 3-11 (1986).

25. Halliwell B., Gutteridge J. M. Free Radicals in Biology and Medicine (Oxford University Press, 2015).

26. Liu, W.-Y., Li, H.-Y., Zhao, B.-X. \& Miao, J.-Y. Synthesis, crystal structure and living cell imaging of a $\mathrm{Cu}^{2+}$-specific molecular probe. Org. Biomol. Chem. 9, 4802-4805 (2011).

27. Hickey, J. L. et al. Intracellular distribution of fluorescent copper and zinc bis (thiosemicarbazonato) complexes measured with fluorescence lifetime spectroscopy. Inorg. Chem. 54, 9556-9567 (2015).

28. Chen, X. et al. Recent progress in the development of fluorescent, luminescent and colorimetric probes for detection of reactive oxygen and nitrogen species. Chem. Soc. Rev. 45, 2976-3016 (2016).

29. Lewis, W. B., Alei, M. \& Morgan, L. O. Magnetic resonance studies on copper (II) complex ions in solution. I. Temperature dependences of the ${ }^{17} \mathrm{O}$ NMR and copper(II) EPR linewidths of $\mathrm{Cu}\left(\mathrm{H}_{2} \mathrm{O}\right)_{6}{ }^{2+}$. J. Chem. Phys. 44, 2409-2417 (1966).

30. Romach, Y. et al. Spectroscopy of surface-induced noise using shallow spins in diamond. Phys. Rev. Lett. 114, 017601 (2015).

31. Lehtinen, O. et al. Molecular dynamics simulations of shallow nitrogen and silicon implantation into diamond. Phys. Rev. B 93, 035202 (2016).

32. Noack, M. \& Gordon, G. Oxygen-17 NMR and copper EPR linewidths in aqueous solutions of copper(II) ion and 2,2'-dipyridine. J. Chem. Phys. $\mathbf{4 8}$ 2689-2699 (1968).
33. Narkowicz, R., Suter, D. \& Stonies, R. Planar microresonators for EPR experiments. J. Magn. Reson. 175, 275-284 (2005).

34. Van Tol, J., Brunel, L.-C. \& Wylde, R. A quasioptical transient electron spin resonance spectrometer operating at 120 and $240 \mathrm{GHz}$. Rev. Sci. Instrum. 76, 074101 (2005).

35. Shtamm, E., Purmal, A. \& Skurlatov, Y. I. Mechanism of catalytic ascorbic acid oxidation system $\mathrm{Cu}^{2+}$-ascorbic acid- $\mathrm{O}_{2}$. Int. J. Chem. Kinet. 11, 461-494 (1979).

36. Acosta, V. M. et al. Diamonds with a high density of nitrogen-vacancy centers for magnetometry applications. Phys. Rev. B 80, 115202 (2009).

37. Yeung, T. K., Le Sage, D., Pham, L. M., Stanwix, P. L. \& Walsworth, R. L. Anti-reflection coating for nitrogen-vacancy optical measurements in diamond. Appl. Phys. Lett. 100, 251111 (2012).

38. Kaviani, M. et al. Proper surface termination for luminescent near-surface NV centers in diamond. Nano Lett. 14, 4772-4777 (2014).

39. Meinhardt, T., Lang, D., Dill, H. \& Krueger, A. Pushing the functionality of diamond nanoparticles to new horizons: orthogonally functionalized nanodiamond using click chemistry. Adv. Funct. Mater. 21, 494-500 (2011).

40. Cong, X., Poyton, M. F., Baxter, A. J., Pullanchery, S. \& Cremer, P. S. Unquenchable surface potential dramatically enhances $\mathrm{Cu}^{2+}$ binding to phosphatidylserine lipids. J. Am. Chem. Soc. 137, 7785-7792 (2015).

41. Rensing, C. \& Grass, G. Escherichia coli mechanisms of copper homeostasis in a changing environment. FEMS Microbiol. Rev. 27, 197-213 (2003).

42. Epstein, R. J., Mendoza, F. M., Kato, Y. K. \& Awschalom, D. D. Anisotropic interactions of a single spin and dark-spin spectroscopy in diamond. Nat. Phys. 1, 94-98 (2005).

\section{Acknowledgements}

The authors acknowledge Drs. Nikolai Dontschuk and Stuart Earl for assistance with the Electron beam lithography. This research was supported in part by the Australian Research Council Centre of Excellence for Quantum Computation and Communication Technology (Project number CE110001027). This work was also supported by the University of Melbourne through the Centre for Neural Engineering and the Centre for Neuroscience. L.C.L.H. acknowledges support of the Australian Research Council under the Laureate Fellowship scheme (FL130100119). S.P. acknowledges the support from the NHMRC Fellowship scheme (1005050). P.S.D. acknowledges the support from the ARC Future Fellowship scheme (FT130100204). S.C.D. acknowledges the support from the ARC Future Fellowship scheme (FT11010019). D.A.S. acknowledges support from the Melbourne Neuroscience Institute Fellowship Scheme.

\section{Author contributions}

D.A.S., L.T.H. and L.C.L.H. conceived the idea of the experiment. D.A.S. designed and constructed the quantum magnetic resonance microscope. R.G.R. and D.A.S. acquired the data with assistance from P.S.D., S.P. and P.M. in transition metal bio-chemistry. R.G.R. developed chemistry protocols and prepared analyte solutions for copper analysis. L.T.H., L.C.L.H., D.A.S. and R.G.R. developed the theoretical framework with input from P.S.D., S.P., S.C.D. and P.M. to describe the results. E.P. fabricated the image mask. S.C. D. performed CW EPR measurements. All authors discussed the results and participated in writing the manuscript.

\section{Additional information}

Supplementary Information accompanies this paper at doi:10.1038/s41467-017-00466-y.

\section{Competing interests: The authors declare no competing financial interests.}

Reprints and permission information is available online at http://npg.nature.com/ reprintsandpermissions/

Publisher's note: Springer Nature remains neutral with regard to jurisdictional claims in published maps and institutional affiliations.

\footnotetext{
Open Access This article is licensed under a Creative Commons Attribution 4.0 International License, which permits use, sharing, adaptation, distribution and reproduction in any medium or format, as long as you give appropriate credit to the original author(s) and the source, provide a link to the Creative Commons license, and indicate if changes were made. The images or other third party material in this article are included in the article's Creative Commons license, unless indicated otherwise in a credit line to the material. If material is not included in the article's Creative Commons license and your intended use is not permitted by statutory regulation or exceeds the permitted use, you will need to obtain permission directly from the copyright holder. To view a copy of this license, visit http://creativecommons.org/ licenses/by/4.0/.
}

(c) The Author(s) 2017 